\title{
INTRAOCULAR TUBERCULOSIS IN A PATIENT WITH ANKYLOSING SPONDYLITIS
}

\author{
Gabriela Stutz Francisco Moreira ${ }^{1, \star}$, Daniella Socci ${ }^{1}$ \\ 1.Hospital Federal dos Servidores do Estado do Rio de Janeiro, Rio de Janeiro (RJ), Brazil. \\ *Corresponding author: gabrielastutz@hotmail.com
}

\section{BACKGROUND}

Spondyloarthritis (SpA) is a chronic inflammatory disease that primarily manifests in the axial skeleton (sacroiliac joints and spine). However, approximately $30 \%$ of patients with axial SpA (axSpA) also have extra-articular manifestations, including psoriasis, inflammatory bowel disease, and acute anterior uveitis. Of these, uveitis is the most common, impacting 25-40\% of patients with SpA uveitis includes a heterogeneous presentation of inflammatory disorders involving the uvea. It can be infectious or noninfectious. Further, uveitis can be subcategorized based on its location within the eye, as anterior, intermediate, posterior or panuveitis. In Brazil, the incidence of tuberculosis is still high. Extrapulmonary disease with ocular manifestation presents a diagnostic challenge due to varied presentation. Intraocular tuberculosis is a great mimicker of various uveitis entities. This ability to mimic other infections is due in part to the location of infection, the host response, and the virulence of the organism. The incidence of tuberculous uveitis (a presumed diagnosis) depends largely upon individual risk factors and the tuberculosis burden of the region. Currently, it is recommended to investigate for ocular TB in any uveitis of unknown etiology, recurrent uveitis or uveitis unresponsive to conventional therapy.

\section{CASE REPORT}

A 44-year-old man, with radiographic axSpA and without extra-articular manifestations, who starts treatment with etanercept, after negative viral serologies (hepatitis and HIV) and nonreactive PPD, came to the office with a complaint of decreased visual acuity. The evaluation with an ophthalmologist revealed anterior uveitis, peripheral retinal vasculitis and the presence of bilateral snowball. At that time, etanercept was suspended and an investigation was started to exclude demyelinating disease, sarcoidosis and pulmonary tuberculosis. The tests for diagnostic investigation were negative, except for PPD, which was 20 mm (strong reactor). At that time, the diagnosis of intraocular tuberculosis was assumed as Possible IOTB (table) and treatment with rifampicin, isoniazid, pyrazinamide and ethambutol was started. After 2 months of treatment, the patient returns to the clinic with a significant improvement in visual acuity.

\section{CONCLUSION}

Brazil is considered to have a high burden of TB, due to its contribution in absolute numbers of tuberculosis cases. In patients with SpA, whose anterior uveitis is an extra-articular manifestation, the diagnosis of intraocular tuberculosis should be kept in mind as differential diagnosis for ocular infection, especially in endemic area, because the earlier it is recognized and the appropriate treatment initiated, the lower the risk of visual morbidity. 